Gazi University
Journal of Science
http://dergipark.gov.tr/gujs

\title{
The Effect of Si (111) Substrate Surface Cleaning on Growth Rate and Crystal Quality of MOVPE Grown AIN
}

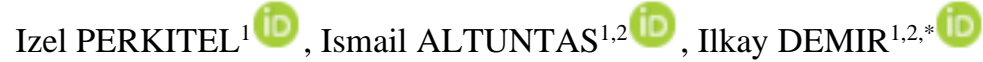 \\ ${ }^{I}$ Nanophotonics Research and Application Center, Sivas Cumhuriyet University, 58140 Sivas, Turkey \\ ${ }^{2}$ Department of Nanotechnology Engineering, Sivas Cumhuriyet University, 58140 Sivas, Turkey \\ Highlights \\ - This paper focuses on the importance of the RCA cleaning process. \\ - Effect of the RCA cleaning process is proposed for on growth rate of AlN layer in the study. \\ - The RCA cleaning process increases the growth rate and improves the crystal quality were obtained.
}

\section{Article Info}

Received: 09 Nov 2020

Accepted: 18 Feb 2021

\begin{tabular}{l} 
Keywords \\
\hline RCA \\
Epitaxial AlN \\
MOVPE \\
X-ray diffraction \\
In situ reflectance
\end{tabular}

\begin{abstract}
In this work, the effect of Si (111) substrate surface cleaning by RCA (Radio Corporation of America) method on growth rate and crystalline quality of epitaxially grown AlN thin films by MOVPE (Metal Organic Vapor Phase Epitaxy) technique is investigated. In situ reflectance system and high resolution X-ray diffraction (HRXRD) technique are used for the analysis of growth rate and crystal quality of epitaxial AlN layers, respectively. Also, The Raman measurement is done to show the effect of the RCA cleaning procedure on the position of the peaks that occurred in the Raman spectra. The results have shown that the surface cleaning of Si (111) substrate by the RCA method removes the oxide layer formed on the surface, also helps to decrease the parasitic reactions and increases the adatom efficiency, results in an increased growth rate of the AlN layer. Besides, surface cleaning of Si (111) substrate by the RCA method has reduced the FWHM value $\sim 5 \%$ for $\omega-2 \theta$ scan and $\sim 60 \%$ for $\omega$ scan of AlN epilayer, indicating an improvement in crystal quality.
\end{abstract}

\section{INTRODUCTION}

Group III-V semiconductors are compounds made by group III elements and group V elements in the periodic table [1]. Compounds ( $\mathrm{GaN}$, InN, AlN, etc.) or alloys (InGaN, AlGaN, InAlGaN, etc.) made by group III elements (In, $\mathrm{Ga}$, and $\mathrm{Al}$ ) and group V element $(\mathrm{N})$ are called III-nitride or III-N semiconductors [2]. III-N semiconductors with wide bandgap and high breakdown voltage have gradually started to replace the traditional materials in the last 20 years. $\mathrm{GaN}$ has been the most popular compound of this group and after obtaining high crystal quality GaN, it was awarded the Nobel Prize in Physics in 2014 for its high brightness blue light emitting diodes (LEDs) studies [3]. It has attracted much attention to the applications of electronic and optoelectronic devices in many fields (daily life, defense industry, health, lighting, and energy) since this award [4]. Optoelectronic devices such as LEDs, laser diodes (LDs), solar cells, photodetectors and electronic devices such as high electron mobility transistors (HEMTs), high power pn diodes, Schottky diodes have been produced using III-N materials in recent years [5, 6]. GaN material has dominated these areas and has gradually become the focus of attention in scientific studies and industry [7]. Almost all LEDs used for high efficiency lighting in the market are GaN based. In 2023, the GaN semiconductor device market is expected to have a market size of $\$ 2.8$ billion [8]. In addition to its excellent properties and success in the lighting industry, GaN crystal has been attracted attention in the high powerfrequency field. There are many Figures of Merit (FOM) compared in the literature in terms of material properties of $\mathrm{Si}$ and $\mathrm{GaN}$, which are dominant in the high power-frequency industry. When the FOM of $\mathrm{GaN}$ is compared to $\mathrm{Si}$ it seems that $\mathrm{GaN}$ has 10 times and 1900 times higher than Si regarding BPFM 
(Bipolar power handling capacity FOM) and FET(Field Effect Transistor) power switching product FOM, respectively [9]. GaN material, instead of entering the market alone with these features, has become interesting for hybrid and electric vehicles, power supplies, and photovoltaic converters of transistors grown on $\mathrm{Si}$ substrate. With the cooperation of $\mathrm{GaN}$ material and the $\mathrm{Si}$ industry $(\mathrm{GaN}$ on $\mathrm{Si}$ ), the market size in the transistor field is expected to be $\$ 600$ million in 2024 and \$ 1.7 billion in 2027 [10].

AlN which is one member of III-N semiconductors has the highest range of the bandgap (6.2 eV) [11], high critical electric field $(450 \mathrm{kV} / \mathrm{cm})$ [12], high thermal conductivity [13], most stable high-temperature performance. AlN is nominated as a candidate material for the near future due to all the excellent properties especially for deep-ultraviolet region and high power-frequency devices. In the last years, it has become interesting thanks to its excellent properties compared to GaN [14]. Due to the extremely high cost of AlN substrate, AlN based optoelectronics and especially electronic applications have not been developed as expected until today. Therefore, AlN-based devices are generally grown on silicon and sapphire substrates. The heteroepitaxial growth of the high crystal quality AlN epitaxial layer on different substrates is one of the most important problems in the literature [15]. AlN layer can be grown more difficult for reasons such as growth at higher temperatures $\left(\sim 1200{ }^{\circ} \mathrm{C}\right)$ compared to $\mathrm{GaN}$ [16], the high parasitic reaction between TMAl $\left(\mathrm{Al}\left(\mathrm{CH}_{3}\right)_{3}\right)$ and $\mathrm{NH}_{3}$ [17], and the low surface mobility of the $\mathrm{Al}$ atom [18]. The parasitic reactions between TMAl and $\mathrm{NH}_{3}$ in the vapor phase are shown below [19]:

$$
\begin{aligned}
\mathrm{Al}\left(\mathrm{CH}_{3}\right)_{3}+\mathrm{NH}_{3} & \rightarrow \mathrm{Al}\left(\mathrm{CH}_{3}\right)_{3} \cdot \mathrm{NH}_{3}, \\
\mathrm{Al}\left(\mathrm{CH}_{3}\right)_{3} . \mathrm{NH}_{3} & \rightarrow \mathrm{Al}\left(\mathrm{CH}_{3}\right)_{2} \cdot \mathrm{NH}_{2}+\mathrm{CH}_{4}, \\
\mathrm{nAl}\left(\mathrm{CH}_{3}\right)_{2} . \mathrm{NH}_{2} & \rightarrow\left[\mathrm{Al}\left(\mathrm{CH}_{3}\right)_{2} . \mathrm{NH}_{2}\right]_{\mathrm{n}} \text { where } \mathrm{n} \geq 3 \\
\mathrm{Al}\left(\mathrm{CH}_{3}\right)_{2} . \mathrm{NH}_{2} & \rightarrow\left[\mathrm{Al}\left(\mathrm{CH}_{3}\right)_{2} . \mathrm{NH}_{2}\right]_{2} \\
& \rightarrow \mathrm{AlNparticles}, \\
{\left[\mathrm{Al}\left(\mathrm{CH}_{3}\right)_{2} . \mathrm{NH}_{2}\right]_{2} } & \rightarrow \text { AlNparticles. }
\end{aligned}
$$

According to Equations (4) and (5), the AlN particles formed during growth are removed from the deposition zone by thermophoresis and do not contribute to growth. The use of silicon substrates is common due to the large-scale use of AlN epitaxial films and the ease of industrial mass production. However, high quality AlN epitaxial films grown on silicon substrates cannot be easily achieved due to the large lattice mismatch and thermal expansion coefficient difference [20], which result in high density defects in AlN epitaxial layers [21, 22]. The silicon substrate surface may also have an impact on AlN epitaxial growth because of chemical contaminants and impurities, which can result in effect the property of grown AlN epitaxial films, device performance, and efficiency [23, 24]. As a result, the silicon substrate surface may be critical regarding epitaxial growth as the silicon surface in the atmospheric environment is naturally oxidized in a short time and affected by organic and inorganic impurities [25]. Radio Corporation of America (RCA) process developed by Werner Kern is a chemical cleaning process applied to clean organic and heavy metal impurities on the substrate. It includes the three main steps; first, cleaning organic impurities. Second, cleaning the layer formed by the self-oxidation of silicon in the atmosphere environment. Last, the removal of ionic (heavy metal residues) impurities [26, 27]. The effects of cleaning the silicon substrate surface from possible impurities such as oxide on the crystal, optical, and electrical properties of the epitaxial film are available in the literature [28]. It is well known that the growth rate of epitaxial growth changes with the growth parameters such as flow rate of metalorganic sources (TMGa $\left(\mathrm{Ga}\left(\mathrm{CH}_{3}\right)_{3}\right)$, TMAl, TMIn $\left(\operatorname{In}\left(\mathrm{CH}_{3}\right)_{3}\right)$, etc.), reactor pressure, and growth temperature [29-32]. However, there is no report in the literature showing the effect of cleaning the surface of the Si (111) substrate on the growth rate of the AIN epitaxial layer to the best of our knowledge.

In this study, the effect of surface cleaning of $\mathrm{Si}$ (111) substrate by RCA process on the growth rate and the structural characterization of MOVPE grown AlN layers is investigated. In-situ reflectance system is used for the analysis of growth rate and high resolution X-ray diffraction (HRXRD) technique is used for the analysis of crystal quality of epitaxial AIN layers. Characterization of epitaxially grown layers can be made by in-situ during growth and ex-situ after growth. In-situ characterization is very effective and also practical since it allows investigating the source of an unknown effect that occurs during the epitaxial growth [33]. For this purpose, a semiconductor laser operating at $880 \mathrm{~nm}$ wavelength is used as an in-situ monitoring 
system to see the growth steps and effects on the AlN layer during growth. Ex-situ characterization technique such as HRXRD is employed to the grown samples to investigate structural characterization. HRXRD measurements are performed by using Rigaku SmartLab diffractometer, equipped with a rotating $\mathrm{Cu}$ anode that provides $9 \mathrm{~kW}$ X-ray power $(45 \mathrm{kV}$ tube voltage and $200 \mathrm{~mA}$ tube current) and a four-bounce Ge (220) monochromator. The Raman spectroscopy-Witec is carried out to make more analysis. In-situ reflectance measurements and XRD scans results have shown that the RCA cleaning procedure increases the growth rate of the AlN layer and improves the crystal quality, respectively.

\section{MATERIAL METHOD}

In this study, two different AlN samples have been grown to investigate the effect of surface cleaning on the growth rate and crystalline quality of AlN. Sample B has been cleaned by the RCA process just before the growth and any cleaning process has been not applied to Sample A. The cleaning procedure is as follows: First step; deionized (DI) water, $\mathrm{H}_{2} \mathrm{O}_{2}, \mathrm{NH}_{4} \mathrm{OH}$ solution in a ratio of 5: 1: 1 is prepared and heated up to $77-80^{\circ} \mathrm{C}$. Before the temperature rises above $80^{\circ} \mathrm{C}$, the substrate is kept in this solution for 12 minutes. The substrate is rinsed with DI water for 1 minute. In this step, a possible oxide layer is removed from the surface. Second step; DI water, $\mathrm{H}_{2} \mathrm{O}_{2}, \mathrm{HCl}$ solution in a ratio of 6: 1: 1 is prepared and heated up to 77$80^{\circ} \mathrm{C}$. Before the temperature rises above $80^{\circ} \mathrm{C}$, the substrate is kept in this solution for 12 minutes. The substrate is rinsed with DI water for 1 minute. In this step, ionic impurities are removed, metal ions and metal hydroxide dissolve using a hydrochloric/peroxide mixture. Third step; DI water, HF solution in a ratio of 100:1 is prepared within a polypropylene beaker. It is immersed in the solution for 20-30 seconds until the substrate is observed to be hydrophobic. The substrate is rinsed with DI water for 1 minute and immersed in another DI water beaker for 2-3 minutes. In this step, the natural oxide is removed from the silicon surface with the aqueous HF solution and the dangling bonds of the silicon are saturated with hydrogen [26, 27]. Then Sample A and B have been grown on a 2 inch (111) oriented Si substrate by AIXTRON 200/4 RF-S horizontal flow MOVPE system.TMAl (trimethylaluminum) precursor is used as a source of $\mathrm{Al}$ (aluminum) and the $\mathrm{N}$ element (nitrogen) is obtained from $\mathrm{NH}_{3}$ (ammonia). Before starting the growth, the $\mathrm{Si}$ substrates have been thermally annealed at $1250{ }^{\circ} \mathrm{C}$ and $100 \mathrm{mbar}$ reactor pressure in $\mathrm{H}_{2}$ ambient for 10 minutes. Then, the reactor temperature and pressure are reduced to $860{ }^{\circ} \mathrm{C}$ and $50 \mathrm{mbar}$, respectively, and the AlN nucleation layer of $\sim 20 \mathrm{~nm}$ thickness has been grown. After the nucleation layer growth, the reactor temperature is increased from $860{ }^{\circ} \mathrm{C}$ to $1370{ }^{\circ} \mathrm{C}$, providing the AlN layer to recrystallize. Subsequently, the AIN layer has been grown by the PALE (pulsed atomic layer epitaxy) technique [34, 35]. Figure 1 shows the schematic representation of grown AlN layers without (a) and with RCA applied (b) Si (111) substrates.

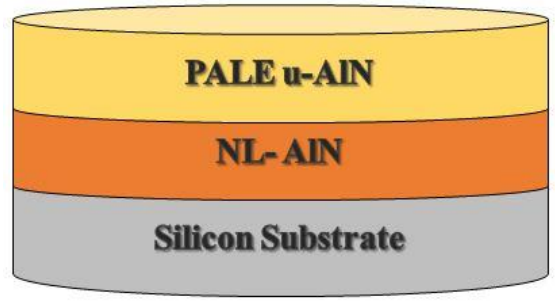

(a)

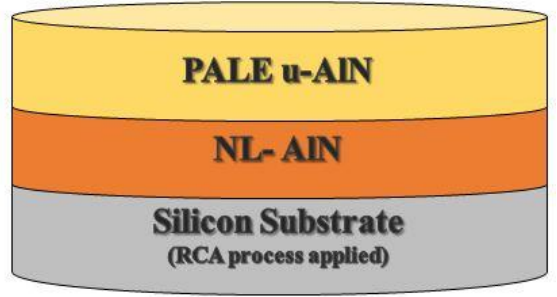

(b)

Figure 1. Schematic diagram of Sample A (a) and Sample B (b)

\section{THE RESEARCH FINDINGS AND DISCUSSION}

Real time in-situ reflectance measurement is a very effective and practical method to observe the grown surface. Figure 2 shows the in-situ reflectance measurements (red and blue curve) and reactor temperature (green curve) of Sample A and Sample B. AlN growth process can be labeled with five steps (i), (ii), (iii), and (iv) together with desorption of silicon substrate. The first step (i) corresponds to the desorption of the silicon substrate. At this step, the substrate is kept at high temperatures $\left(1250{ }^{\circ} \mathrm{C}\right)$ and under hydrogen ambient to remove impurities on the surface. The second step (ii) corresponds to the growth of the low temperature $\left(860^{\circ} \mathrm{C}\right)$ AlN nucleation layer. The third step (iii) corresponds to the recrystallization step. At this step, the reactor temperature is increased and the AlN layer grown at low temperature is recrystallized. 
The fourth step (iv) corresponds to high temperature $\left(1370{ }^{\circ} \mathrm{C}\right)$ AlN growth. Since AlN has a smaller refractive index than silicon [36, 37], the reflectance shows a decreasing behavior. It is seen from Figure 2 that the finish points of reflectance curves of Sample A and B are different which proves that surface cleaning of $\mathrm{Si}$ (111) substrate changes the growth rate of epitaxially grown AlN.

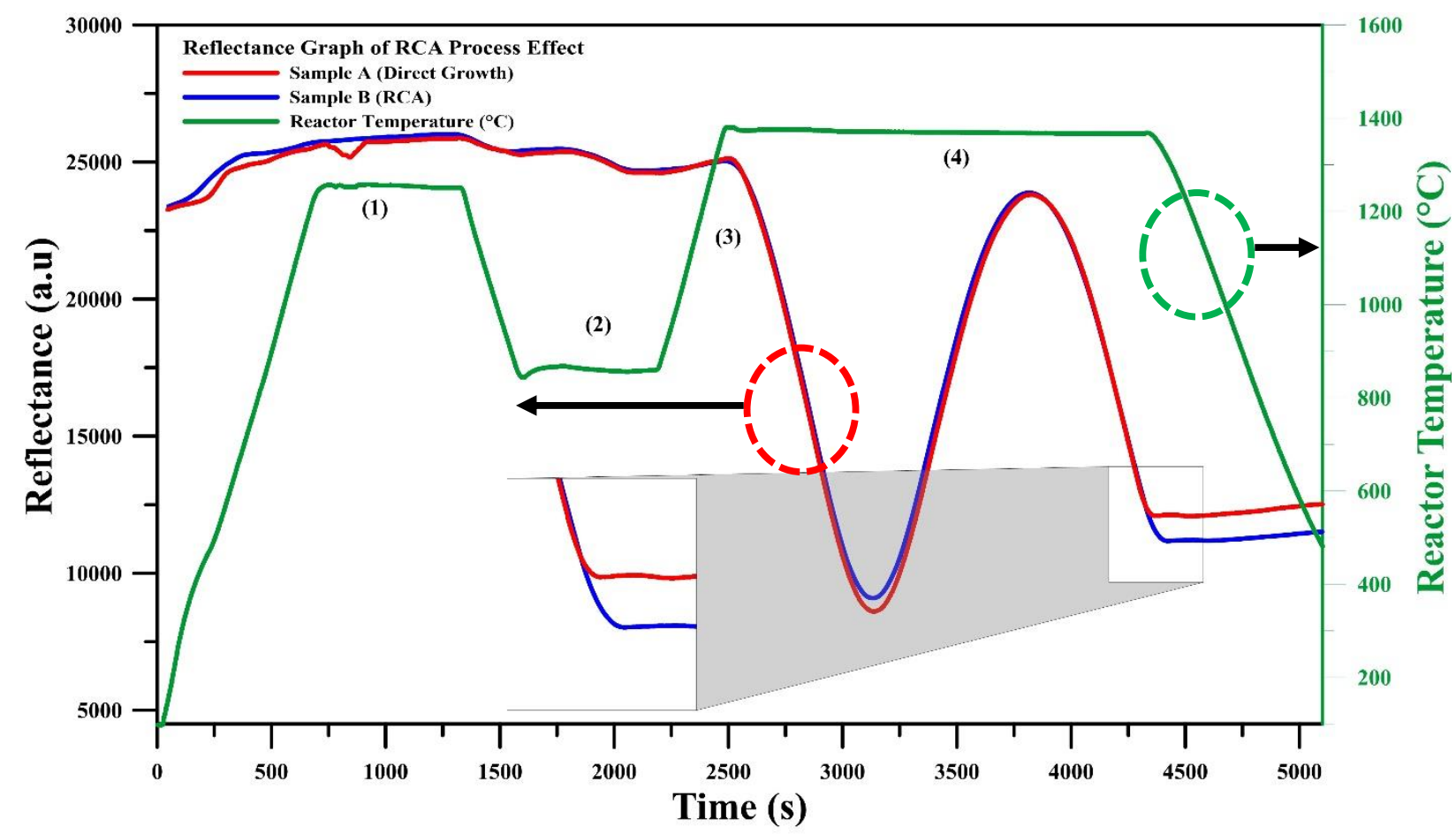

Figure 2. In situ reflectance measurements (red and blue curve) of Sample A and Sample B and reactor temperature (green curve)

Figure 3 shows the growth rates of Sample A and Sample B calculated from in situ reflectance measurements. The growth rate of the AlN layer grown on Si substrate with RCA has been obtained as $0.162 \mathrm{~nm} / \mathrm{s}$, and the growth rate of the AlN layer grown on Si substrate without RCA has been obtained as $0.158 \mathrm{~nm} / \mathrm{s}$. It is concluded from the observations and calculations that the only reason for the growth rate difference between the two samples is surface cleaning. It is also thought that the surface cleaning by the RCA process removed as much as the oxide layers and it helps to decrease the parasitic reactions and increase the adatom efficiency to produce more AlN epitaxial films.

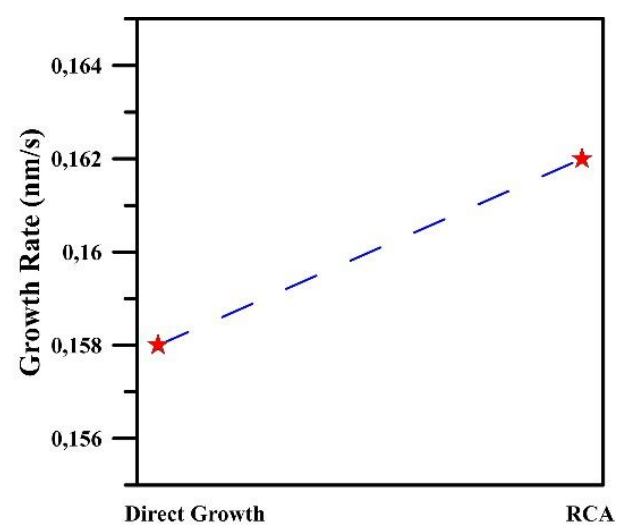

Figure 3. Effect of the RCA process on the growth rate 
HRXRD technique is used for the analysis of the crystalline quality of epitaxial AlN layers. $\omega-2 \theta$ and $\omega$ scans give information about the lateral and vertical crystalline quality and the interface transition roughness of the crystal structures. In these scans, the peak intensity and FWHM value are related to the crystal quality. The FWHM value gives information about the stress occurring in the structure. As the stress increases, the FWHM value increases linearly. Also, the defect density is inversely proportional to the peak intensity [38]. As it is known different types of stresses exist in materials. First-order stresses, called macrostresses, are homogeneous on a large scale, involving many grains (i.e., a few hundred microns). The fact that these stresses shift the peak position is the main effect on the XRD peak. The second and third-order stresses (microstresses) are homogeneous at the size of about tens of microns (small areas like part of a grain). Second and third order stresses manifest with the broadening of the XRD peak, i.e., the increase in FWHM [39].

In a $\omega-2 \theta$ scan, the $x$-ray source, and the detector are moved the same amount relative to the sample surface around the determined Bragg angle. Meanwhile, the momentum transition vector is moved vertically to the relevant crystal planes of the sample. Thus, the vertical lattice constant, roughness between vertical planes, grain size analysis, strain, and stress analysis, etc. can be obtained from the $\omega-2 \theta$ scan. Figure 4 shows the $\omega-2 \theta$ scans of Sample A and Sample B. Also, the FWHM values and the peak intensity of the AlN peaks have been compared in the inset of the graph. Sample A has a FWHM value of 996 arcsec and a maximum intensity of 227 a.u. Sample B has a FWHM value of 944 arcsec and a maximum intensity of 1670 a.u. In other words, Sample B has not only a lower FWHM value but also a high peak intensity. Therefore, an improvement in the crystal quality is observed with the decrease of the stress in the structure.

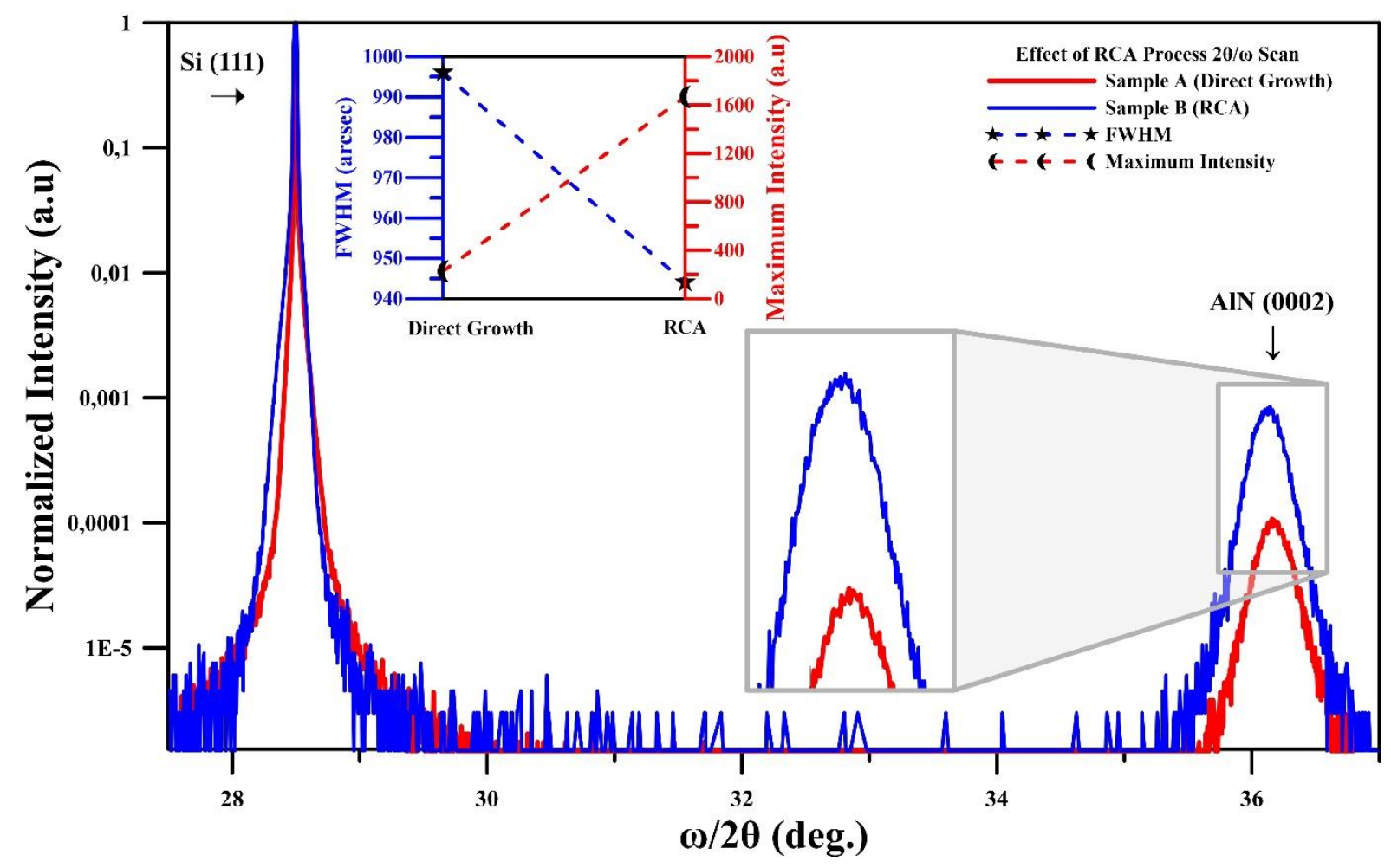

Figure 4. HRXRD $\omega-2 \theta$ scans of Sample A and Sample B

The c-axis lattice constants of the AlN films have been deduced from the $\omega$ - $2 \theta$ scans for Sample A and B. The Bragg's angle of high angular reflection (0002) which corresponds to the AlN film has been utilized to determine the lattice spacing $\left(\mathrm{d}_{\mathrm{hkl}}\right)$ from Bragg's law. The lattice parameters and out-of-plane residual strain for hexagonal crystal have been calculated using the following equations [40]: 
$\frac{1}{d_{h k l}^{2}}=\frac{4}{3}\left(\frac{h^{2}+k^{2}+h k}{a^{2}}\right)+\frac{l^{2}}{c^{2}}$ and $\varepsilon_{\mathrm{zz}}=\frac{\mathrm{c}-c_{0}}{c_{0}}$

where $\mathrm{h}, \mathrm{k}$, and 1 are miller indices, the lattice parameter $\mathrm{c}_{0}$ of $0.4978 \mathrm{~nm}$ corresponds to the bulk AlN crystal and $\varepsilon_{z z}$ is an out-of-plane residual strain. The c-axis lattice constants (out-of-plane residual strain) for Sample A and B have been calculated $0.496851 \mathrm{~nm}(0.002465)$ and $0.497477 \mathrm{~nm}(0.00121)$, respectively.

In general, another important scan to investigate the quality of semiconductor crystals is the $\omega$-scan. In $\omega$ scan, after the Bragg angle of the relevant plane of the sample is found, the x-ray source and the detector are moved in the same direction. Thus, the magnitude of the momentum transition vector is constant in the vertical direction. By changing the momentum transition vector in the horizontal direction, the quality of the crystal in the plane and the interface effect can be obtained. Figure 5 shows the $\omega$-scans of the two samples. When the peak FWHM of the AIN peaks have been compared, it is clear that Sample A has a larger FWHM than Sample B. Sample A has a FWHM value of 18273 arcsec and a maximum intensity is 227 a.u. while Sample B has a FWHM value is 6983 arcsec and maximum intensity of 1649 a.u. Therefore, it is clearly shown that when the RCA cleaning process is applied the crystal quality of epitaxial AlN improves.

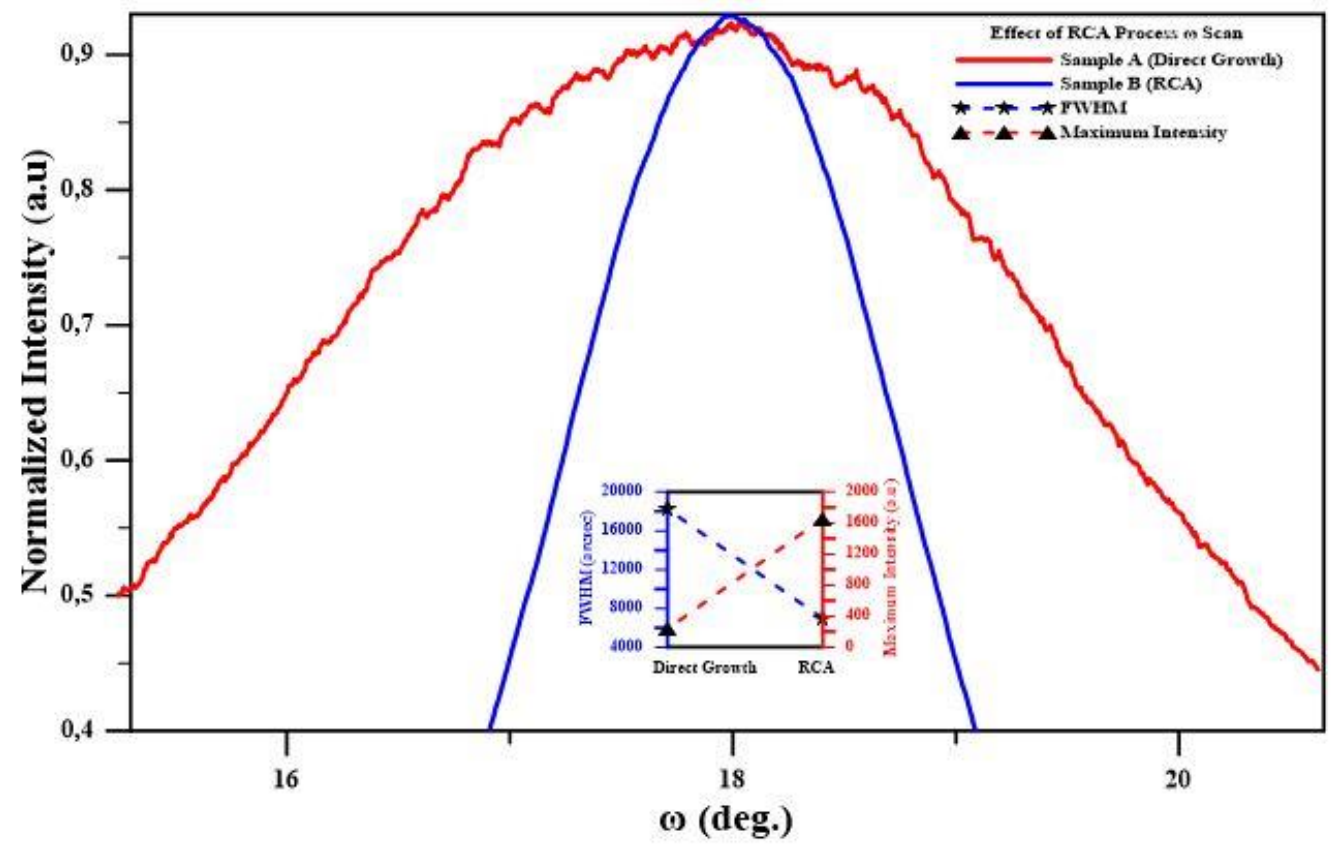

Figure 5.Normalized HRXRD (0002) $\omega$-scans of Sample A and Sample B

The natural oxide layer on the Si substrate leads to low coherence between the AlN layer and the Si substrate. Also, since the interfacial layer is amorphous it is very difficult to grow epitaxial AlN on top of this amorphous layer. This makes it difficult to obtain a smooth AlN surface for the growth of AlN on the Si substrate [41]. A smooth, oxide-free Si surface is essential for the growth of a quality AlN layer. The pre-growth Si surface is very important in determining crystal quality and thus the stress of AlN [42]. As a result, the removal of the oxide layer on the Si surface decreases the tensile stress in the structure. Thus, the decrease in tensile stress in the AlN causes a decrease in FWHM.

The Raman spectra of Sample A and B are demonstrated in Figure 6. The Raman spectra for Sample A and B exhibit the peaks of $E_{2}$ (high) of 652.7 and $653.8 \mathrm{~cm}^{-1}, \mathrm{~A}_{1}(\mathrm{TO})$ of 619.2 and $618.0 \mathrm{~cm}^{-1}$, and $\mathrm{A}_{1}(\mathrm{LO})$ of 881.7 and $883.9 \mathrm{~cm}^{-1}$, respectively. The Raman spectra of the bulk AlN crystal give the $\mathrm{E}_{2}(\mathrm{high}), \mathrm{A}_{1}(\mathrm{TO})$, and $\mathrm{A}_{1}(\mathrm{LO})$ at 657,610 , and $890 \mathrm{~cm}^{-1}$, respectively [43]. 


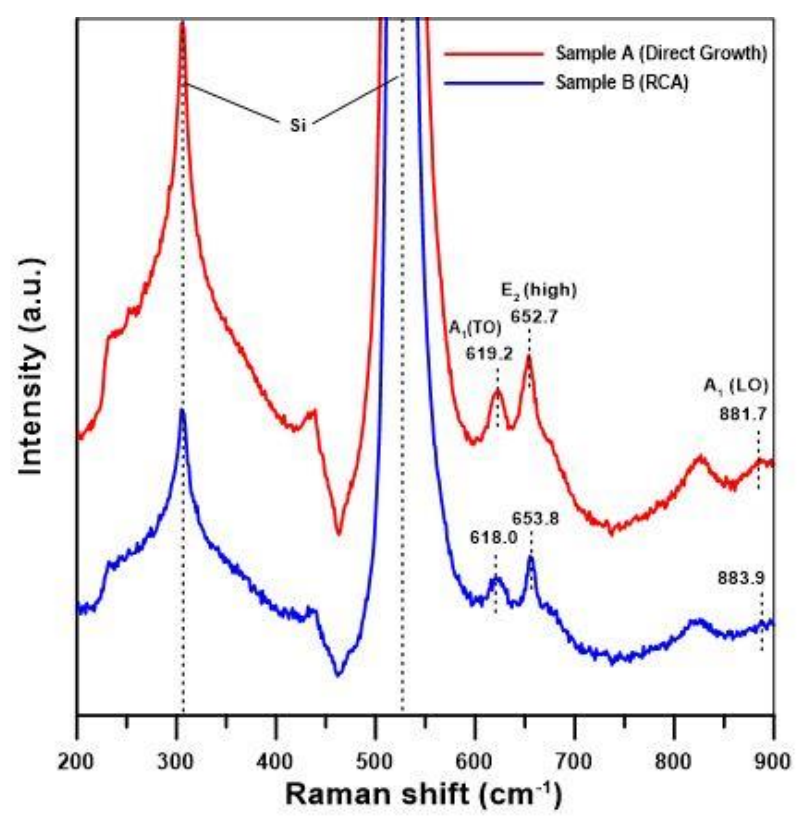

Figure 6. Raman spectra of Sample A and B at room temperature

The shift in $\mathrm{E}_{2}$ (high) peak frequency has been seen in Figure 6 resulting from the strain. The wavenumber of the $\mathrm{E}_{2}$ (high) mode is $652.7 \mathrm{~cm}^{-1}$ for Sample A, while it is $653.8 \mathrm{~cm}^{-1}$ for Sample B. The Raman peak shifts to a higher frequency due to compression stress and to a lower frequency due to tensile stress [44]. It seems that the wavenumber of the $E_{2}$ (high) shifts almost $4.3 \mathrm{~cm}^{-1}$ and $3.2 \mathrm{~cm}^{-1}$ which corresponds to the existence of the tensile strain for Sample A and B, respectively. So, it is understood that Sample A has a larger tensile strain than that of Sample B. Also, in-plane tensile stresses and strains for Sample A and B have been calculated by using the following equations:

$\sigma=\frac{\text { phonon peak shift }}{\mathrm{K}} \quad$ and $\quad \varepsilon=\frac{\sigma}{\gamma}$

where $\mathrm{K}$ is the pressure coefficient of $4.3 \mathrm{~cm}^{-1} \mathrm{GPa}^{-1}, \sigma$ is the in-plane tensile stress and $\Upsilon$ is the biaxial stress of AlN of $469 \mathrm{GPa}[45,46]$. The values of in-plane stress (strain) have been obtained $1 \mathrm{GPa}(0.00213)$ and $0.74 \mathrm{GPa}(0.00158)$ for Sample A and B, respectively.

\section{RESULTS}

In this study, the effect of surface cleaning of $\mathrm{Si}$ (111) the substrate by RCA process on the growth rate and the structural characterization of MOVPE grown AlN layers is investigated. In-situ reflectance system and HRXRD technique are used for the analysis of growth rate and crystalline quality of epitaxial AlN layers, respectively. It has been shown that the RCA process increases the growth rate of AlNepilayer from 0.158 $\mathrm{nm} / \mathrm{s}$ to $0.162 \mathrm{~nm} / \mathrm{s}$. Even the growth rates look similar since one of the main obstacles of the commercial development of AlN is its low growth rate, it is thought that a small growth rate difference of Sample A and B should be taken into consideration. It is thought that the RCA process removes oxide layers as much as possible, thus helping to reduce parasitic reactions. When surface cleaning of $\mathrm{Si}$ (111) is applied, it has been observed that the FWHM values of AlN of $\omega-2 \theta$ scan decrease from 996 arcsec to 944 arcsec and the maximum intensities of AlN in $\omega$-2 $2 \theta$ scan increase from 227 a.u. to 1640 a.u. Also, it is observed that the FWHM values of AlN of $\omega$ scan decrease from $18273 \operatorname{arcsec}$ to $6983 \mathrm{arcsec}$, and the maximum intensities of AlN of $\omega$ scan increase from 227 a.u to 1649 a.u. Therefore, it is clearly shown that the RCA cleaning procedure improves the crystalline quality by decreasing the stress in the structure. 


\section{CONFLICTS OF INTEREST}

No conflict of interest was declared by the authors.

\section{ACKNOWLEDGMENTS}

This study is supported by TUBITAK under the project numbers 117F339, 118F425 and by the Scientific Research Project Fund of Sivas Cumhuriyet University under the Project number M-772. The authors acknowledge the usage of the Nanophotonics Research and Application Center at Sivas Cumhuriyet University (CUNAM) facilities.

\section{REFERENCES}

[1] Morkoç, H., Nitride Semiconductor Devices: Fundamentals and Applications, Wiley, (2013).

[2] Kolluri, S., Pei, Y., Keller, S., Denbaars, S. P., Mishra, U. K., "RF performance of N-polar $\mathrm{AlGaN} / \mathrm{GaN}$ MIS-HEMTs grown by MOCVD on sapphire substrate", IEEE Electron Device Letters, 30(6): 584-586, (2009).

[3] Cai, Q., Ge, M., Xue, J., Hu, L., Chen, D., Lu, H., Zhang, R., Zheng, Y., “An improved design for solar-blind AlGaN avalanche photodiodes", IEEE Photonics Journal, 9(4): 1-7, (2017).

[4] Demir, I., Altuntas, I., Kasapoğlu, A., Mobtakeri, S., Gur, E., Elagoz, S., "Microstructural evolution of MOVPE grown GaN by the carrier gas", Semiconductors, 52(16): 2030-2038, (2018).

[5] Okumura, H., Suihkonen, S., Lemettinen, J., Uedono, A., Zhang, Y., Piedra, D., Palacios, T., “AlN metal-semiconductor field-effect transistors using Si-ion implantation", Japanese Journal of Applied Physics, 57(4S): 04FR11, (2018).

[6] Wierer, Jr. JJ, Tsao, J.Y., Sizov, D.S., "The potential of III-nitride laser diodes for solid-state lighting”, Physica Status Solidi (c), 1(3-4): 674-677, (2014).

[7] Altuntas, I., Demir, I., Kasapoğlu, A.E., Mobtakeri, S., Gur, E., Elagoz, S., "The effects of twostage HT-GaN growth with different V/III ratios during 3D-2D transition", Journal of Physics D: Applied Physics, 51(3): 035105, (2017).

[8] https://compoundsemiconductor.net/article/103024/GaN_device_Market_Worth_over_34_Billio n_by_2024. Access date: 08.12.2017.

[9] Ozpineci, B., Tolbert, L.M., "Comparison of wide-bandgap semiconductors for power electronics applications", United States, Department of Energy, (2004).

[10] https://compoundsemiconductor.net/article/102889/GaN_Device_Market_To_Reach_2247_Billi on_By_2023\%7BfeatureExtra\%7D. Access date: 10.11.2017

[11] Magnuson, M., Mattesini, M., Höglund, C., Birch, J., Hultman, L., "Electronic structure and chemical bonding anisotropy investigation of wurtzite AlN", Physical Review B, 80(15): 155105, (2009).

[12] O'Leary, S.K., Siddiqua, P., Hadi, W.A., Foutz, B.E., Shur, M.S., Eastman, L.F., "Electron transport within III-V nitride semiconductors", In Springer Handbook of Electronic and Photonic Materials, 1-1, Springer, Cham, (2017).

[13] Cheng, Z., Koh, Y.R., Mamun, A., Shi, J., Bai, T., Huynh, K., Yates, L., Liu, Z., Li, R., Lee, E., Liao, M. E., Wang, Y., Yu, H. M., Kushimoto, M., Luo, T., Goorsky, M. S., Hopkins, P. E., 
Amano, H., Khan, A., Graham, S., "Experimental observation of high intrinsic thermal conductivity of AlN", Physical Review Materials, 4(4): 044602, (2020).

[14] Young, E.C., Yonkee, B.P., Wu, F., Oh, S.H., DenBaars, S.P., Nakamura, S., Speck, J. S., "Hybrid tunnel junction contacts to III-nitride light-emitting diodes", Applied Physics Express, 9(2): 022102, (2016).

[15] Demir, I., Li, H., Robin, Y., McClintock, R., Elagoz, S., Razeghi, M., "Sandwich method to grow high quality AlN by MOCVD”, Journal of Physics D: Applied Physics, 51(8): 085104, (2018).

[16] Yan, J., Wang, J., Zhang, Y., Cong, P., Sun, L., Tian, Y., Zhao, C., Li, J., “AlGaN-based deepultraviolet light-emitting diodes grown on high-quality AlN template using MOVPE", Journal of Crystal Growth, 414: 254-257, (2015).

[17] Creighton, J. R., Wang, G. T., Breiland, W. G., Coltrin, M. E., "Nature of the parasitic chemistry during AlGaInN OMVPE”, Journal of Crystal Growth, 261(2-3): 204-213, (2014).

[18] Wang, T.Y., Liang, J.H., Fu, G.W., Wuu, D.S., "Defect annihilation mechanism of AlN buffer structures with alternating high and low V/III ratios grown by MOCVD”, CrystEngComm, 18(47): 9152-9159, (2016).

[19] Zhao, D., Zhu, J., Jiang, D., Yang, H., Liang, J., Li, X., Gong, H. M., "Parasitic reaction and its effect on the growth rate of AlN by metalorganic chemical vapor deposition", Journal of Crystal Growth, 289(1): 72-75, (2006).

[20] Demir, I,, Robin, Y., McClintock, R., Elagoz, S., Zekentes, K., Razeghi, M., "Direct growth of thick AIN layers on nanopatterned Si substrates by cantilever epitaxy", Physica Status Solidi (a), 214(4): 1600363, (2017).

[21] Kukushkin, S., Osipov, A., Sergeeva, O., Kiselev, D., Bogomolov, A., Solnyshkin, A., Kaptelov, E. Y., Senkevich, S. V., Pronin, I. P., "Pyroelectric and piezoelectric responses of thin AlN films epitaxy-grown on a SiC/Si substrate", Physics of the Solid State, 58(5): 967-970, (2016).

[22] Kurose, N., Ozeki, K., Araki, T., Iwata, N., Kamiya, I., Aoyagi, Y., "Realization of conductive AlN epitaxial layer on Si substrate using spontaneously formed nano-size via-holes for vertical AlGaN high power FET", Compound Semiconductor Week (CSW) [Includes 28th International International Conference on Indium Phosphide \& Related Materials (IPRM) \& 43rd International Symposium on Compound Semiconductors (ISCS), IEEE, (2016).

[23] Huck, L. A., Buriak, J. M., "Toward a mechanistic understanding of exciton-mediated hydrosilylation on nanocrystalline silicon", Journal of the American Chemical Society, 134(1): 489-497, (2012).

[24] Kern, W., "Overview and evolution of silicon wafer cleaning technology", Handbook of Silicon Wafer Cleaning Technology: Elsevier, 3-85, (2018).

[25] Lafatzis, D., Mergia, K., "Oxidation behaviour of Si wafer substrates in air", Journal of Applied Physics, 114(14): 144308, (2013).

[26] Bera, B., "Silicon wafer cleaning: a fundamental and critical step in semiconductor fabrication process", International Journal of Applied Nanotechnology, 5(1): 8-13, (2019).

[27] Zhao, M. Y., Self-aligned polysilicon gate metal-oxide-semiconductor field effect transistor for large area electronics, Doctoral dissertation, School of Engineering Science-Simon Fraser University, (2005). 
[28] Huang, L., Li, Y., Wang, W., Li, X., Wang, H., Zhang, Z., Li, G., "Growth of high-quality AlN epitaxial film by optimizing the Si substrate surface", Applied Surface Science, 435: 163-169, (2018).

[29] Buegler, M., Gamage, S., Atalay, R., Wang, J., Senevirathna, M., Kirste, R., Xu, T., Jamil, M., Ferguson, I., Tweedie, J., Collazo, R., Hoffmann, A., Sitar, Z., Dietz, N., "Growth temperature and growth rate dependency on reactor pressure for InN epilayers grown by HPCVD", Physica Status Solidi (c), 8(7-8): 2059-2062, (2011).

[30] Demir, I., Elagoz, S., "V/III ratio effects on high quality InAlAs for quantum cascade laser structures", Superlattices and Microstructures, 104: 140-148, (2017).

[31] Meng, J., Jaluria, Y., "Numerical Simulation of GaN Growth in a MOCVD Process", ASME International Mechanical Engineering Congress and Exposition, (2011).

[32] Ni, J., Hao, Y., Zhang, J., Yang, L., "Effect of reactor pressure on the growth rate and structural properties of GaN films", Chinese Science Bulletin, 54(15): 2595-2598, (2009).

[33] Demir, I., Altuntas, I., Bulut, B., Ezzedini, M., Ergun, Y., Elagoz, S., "Comprehensive growth and characterization study on highly n-doped InGaAs as a contact layer for quantum cascade laser applications", Semiconductor Science and Technology, 33(5): 055005, (2018).

[34] Demir, I., Kocak, Y., Kasapoğlu, A.E., Razeghi, M., Gur, E., Elagoz, S., “AlGaN/AlN MOVPE heteroepitaxy: pulsed co-doping $\mathrm{SiH}_{4}$ and TMIn", Semiconductor Science and Technology, 34(7): 075028, (2019).

[35] Zhang, J., Kuokstis, E., Fareed, Q., Wang, H., Yang, J., Simin, G., Khan, M., A, "Pulsed atomic layer epitaxy of quaternary AlInGaN layers", Applied Physics Letters, 79(7): 925-927, (2001).

[36] Adachi, S., Optical Constants of Crystalline and Amorphous Semiconductors: Numerical Data and Graphical Information, Springer Science \& Business Media, (2013).

[37] Schinke, C., Christian Peest, P., Schmidt, J., Brendel, R., Bothe, K., Vogt, M. R., Kröger, I., Winter, S., Schirmacher, A., Lim, S., Nguyen, H. T., MacDonald, D., "Uncertainty analysis for the coefficient of band-to-band absorption of crystalline silicon", AIP Advances, 5(6): 067168, (2015).

[38] Elagoz, S., Demir, I., "Growth of InGaAs/InAlAs superlattices by MOCVD and precise thickness determination via HRXRD”, Gazi University Journal of Science, 29(4): 947-951, (2016).

[39] Marciszko, M., "Diffraction study of mechanical properties and residual stresses resulting from surface processing of polycrystalline materials", Ecole nationale supérieure d'arts et métiersENSAM; AGH University of Science and Technology, Cracovie, Pologne, (2013).

[40] Nilsson, D., Janzén, E., Kakanakova-Georgieva, A., "Lattice parameters of AlN bulk, homoepitaxial and heteroepitaxial material", Journal of Physics D: Applied Physics, 49(17): 175108, (2016).

[41] Tran, B.T., Maeda, N., Jo, M., Inoue, D., Kikitsu, T., Hirayama, H., "Performance improvement of AlN crystal quality grown on patterned Si (111) substrate for deep UV-LED applications", Scientific Reports, 6(1): 1-6, (2016). 
[42] Chandrasekar, H., Mohan, N., Bardhan, A., Bhat, K., Bhat, N., Ravishankar, N., Raghavan, S., "An early in-situ stress signature of the AlN-Si pre-growth interface for successful integration of nitrides with (111) Si”, Applied Physics Letters, 103(21): 211902, (2013).

[43] Altuntas, I., Kocak, M.N., Yolcu, G., Budak, H.F., Kasapoğlu, A.E., Horoz, S., Gur,E., Demir, I., "Influence of the PALE growth temperature on quality of MOVPE grown AlN/Si (111)", Materials Science in Semiconductor Processing, 127: 105733, (2021).

[44] Trodahl, H., Martin, F., Muralt, P., Setter, N., "Raman spectroscopy of sputtered AlN films: E2 (high) biaxial strain dependence", Applied Physics Letters, 89(6): 061905, (2006).

[45] Abd Rahman, M.N., Talik, N.A., Khudus, M.I.A., Sulaiman, A.F., Allif, K., Zahir, N.M., Shuhaimi, A., "Ammonia flux tailoring on the quality of AlN epilayers grown by pulsed atomiclayer epitaxy techniques on (0001)-oriented sapphire substrates via MOCVD", CrystEngComm, 21(12): 2009-2017, (2019).

[46] Wagner, J.M., Bechstedt, F., "Electronic and phonon deformation potentials of GaN and AlN: ab initio calculations versus experiment", Physica Status Solidi (b), 234(3): 965-969, (2002). 